\title{
Understanding Undergraduate Students' Experiences of Telepresence Robots on Campus
}

\author{
Negar Khojasteh \\ nk586@cornell.edu \\ Cornell University \\ Ithaca, NY
}

Susan R. Fussell

sfussell@cornell.edu

Cornell University

Ithaca, NY

\author{
Cathy Liu \\ mcl247@cornell.edu \\ Cornell University \\ Ithaca, NY
}

\section{ABSTRACT}

Mobile robotic telepresence systems (MRPs) allow users to have a video conferencing communication channel with people in a distant environment where the MRP is physically located. In addition to having an audiovisual channel, the remote user can navigate the MRP. In this study, we interviewed ten undergraduate students after they had conducted a search-and-find task using an MRP. We found that students find it easy to maneuver the MRP and value the ability to move around in a remote space as well as having a physically embodied representation. We also found that students face challenges in approaching people due to three reasons, self-presentational concerns, novelty of robot-mediated communication, and difficulty with interpreting nonverbal cues. We will discuss our findings and provide design suggestions that could make MRPs more useful for social interactions.

Permission to make digital or hard copies of part or all of this work for personal or classroom use is granted without fee provided that copies are not made or distributed for profit or commercial advantage and that copies bear this notice and the full citation on the first page. Copyrights for third-party components of this work must be honored. For all other uses, contact the owner/author(s)

CSCW'19 Companion, November 9 - 13, 2019, Austin, TX, USA

(c) 2019 Copyright held by the owner/author(s).

ACM ISBN 978-1-4503-6692-2/19/11. https://doi.org/10.1145/3311957.3359450. 


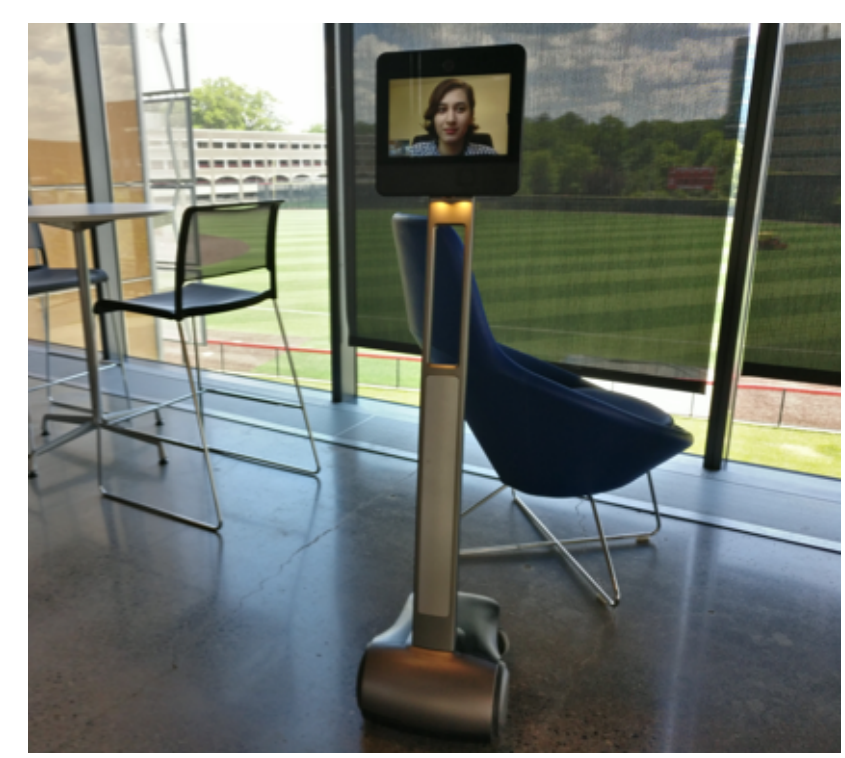

Figure 1: The MRP used in this study, the Beam. The screen shows remote user.

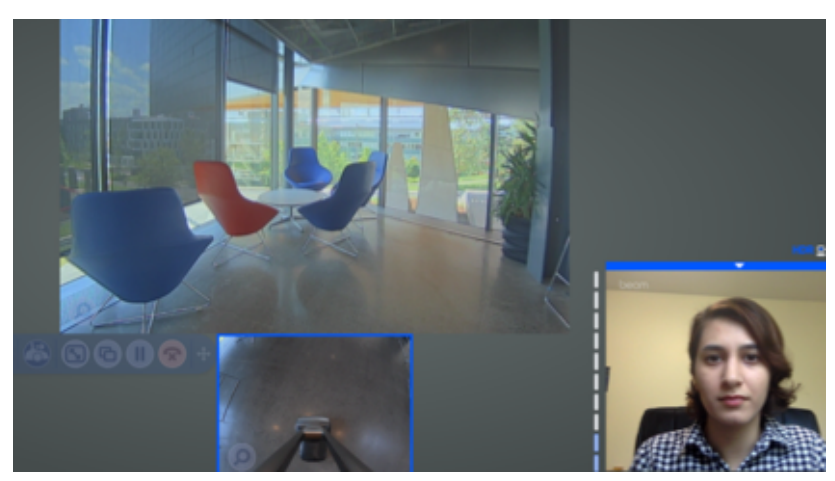

Figure 2: Participants' view of the remote location via beam software

\section{INTRODUCTION}

Mobile robotic telepresence systems (MRPs) are a relatively new group of technologies that provide an audiovisual channel of communication over a mobile base that is controlled by a user in a different location. Using MRPs, users (the pilots) can move around and have conversations with people (the locals) in the distant location collocated with the MRP system [1](Figs. 1 and 2). The benefits and challenges associated with the unique features of MRP, physical embodiment and mobility, which distinguishes them from conventional computer mediated communication tools (e.g., video chat apps), have been studied by researchers in various contexts such as for remote conference attendance $[8,13]$, distant collaboration (e.g., [14, 19]) and remote classroom attendance (e.g., [9]).

One advantage of MRP tools is the ability to move around in another environment, which allows for impromptu, informal interaction $[6,8]$ with people in the remote environment. This has benefits in various contexts (e.g., [5]) one of which is remote education. Students might need to miss school for various reasons (e.g., sickness) which could result in missing social connection with other students and teachers. Previous work has shown the positive role that MRPs could have in remote education $[2,4,9,10,18]$. College students who might likewise be unable to attend school due to reasons such as sickness could benefit from the use MRPs for attending school remotely. Inspired by research mentioned above about remote education, we aim to look at use of MRPs by college students. More specifically, this work aims to contribute to current research on remote education with focusing on challenges students face with initiating conversations with local people.

Findings of this study shows that students value the mobility and physical embodiment that MRPs afford, but they find it hard to approach people to initiate conversations due to concerns around self-presentation and difficulty in interpreting local people's nonverbal behavior. We will conclude with discussion and design suggestions for making MRPs more suitable for informal interactions.

\section{METHODS}

We recruited 10 (6 female, 4 male) undergraduate students for this study. They came to the lab, signed a consent form approved by IRB, were instructed on how to use the MRP, completed a search task and were interviewed regarding their experience. Participants were asked to use a Beam+ telepresence robot [15] to find a printer located in one of the hallways and a specific room which was located in the same building floor (Fig 1 and 2). They were told that they could ask anyone in the space for help with task and were encouraged to interact with local people. We chose this task as a type of task that student might want to do outside class in order to find professors' offices, group study sessions, etc. In all sessions, the robot speed was set to its maximum speed of Beam (1 mph). Upon completion of the task we conducted semi-structured interviews. Audio files of interviews were transcribed verbatim and were coded by two members of the research team following an open coding process [7]. Codes were discussed, similar codes were merged and based on 24 codes we defined main themes and sub-themes. 


\section{Quote 1.}

"I noticed that I was on the fastest speed, but it still wasn't that fast. Yeah. I think like the slowness is good for safety. But sometimes you just want to go straight and there's a lot of space and no one's there, you want to just travel faster." (P6, female)

\section{Quote 2.}

"with Zoom, it is more simple, and this [Beam] is more realistic, with beam, you can physically be there because the machine is representing your physical being by helping you to do what you want and move around" (P5, male)

\section{Quote 3.}

"I think face to face I would have felt more comfortable interrupting people, like hey do you have a minute. But here I'm not sure what the other person is seeing or how much of me they see so I was a little wary of even just interacting in the first place." (P10, female)

\section{Quote 4.}

"People might be surprised talking to like a robot or something. They would not expect a robot suddenly speaking. Yeah, that's why I was kind of hesitant." (P3, female)

\section{Quote 5.}

"[compared to Beam] I feel like in person there's more, there's a mutual understanding of body language and you can read other people or personally, I'm able to read how people act or if they want to talk to me or if they don't." (P7, male)

\section{FINDINGS}

We found two main themes based on our qualitative analysis of the interviews: personal aspects of MRP use and interpersonal aspects of MRP use, which are explained below.

\section{Personal Aspects of MRP Use}

All of our participants said that they enjoyed their experience and majority of them (9 of 10) said that controlling the robot was relatively easy and that they would be willing to use the MRP to attend school during the times that they cannot attend in person. While previous work suggests that driving the robot could be difficult for first time MRP users (e.g., [8]), at least in the context of our study and with young adults as remote users, the majority of the participants reported that control of the Beam+ was easy.

Our observation also shows that people were able to drive the robot smoothly with no collisions in most cases during driving sessions that lasted 15 minutes on average. However, several participants mentioned that the robot seemed to be moving slowly (see Quote 1). More than half of the participants (7 of 10) mentioned the physical embodiment and mobility of the MRP as useful features and some participants considered it an advantage of the MRP over other video conferencing tools (see Quote 2).

\section{Interpersonal Aspects of MRP Use}

During all sessions, we noticed that engaging in social interaction with local people was not common and participants were hesitant to approach local people to ask for help even when they were struggling to complete the search-and-find tasks. We asked them about this and based on their responses we identified three reasons underlying this issue: self-presentational concerns, novelty of robot-mediated communication, and difficulty interpreting nonverbal cues.

Self-presentational concerns. Some of our participants (4 of 10) reported concerns related to self-presentation and impression management which is consistent with research on MRP $[8,16]$. They pointed out concerns about how they looked and sounded to local people as a reason for limited social interactions. (see Quote 3)

Novelty of robot-mediated communication. Three out of 10 participants mentioned that they felt uncomfortable approaching others while using the robot and that talking via MRP felt strange to them. Since all participants were first time users of an MRP, this may be due to the novelty of the MRP and might be alleviated by repeated use and gaining of experience. (see Quote 4)

Difficulty interpreting nonverbal signals. Half of the participants talked about challenges in interpreting locals' status (e.g., busy or not busy) as well as their nonverbal signals such as interest in initiating a conversation. In face to face interactions, nonverbal cues such as eye contact help people with interpersonal communication. However, when people use CMC tools, interpreting and signaling mutual attention could be challenging. (see Quote 5) 


\section{Limitations.}

This paper presents the preliminary findings of an exploratory study and has limitations. Participants performed only one type of task, search and find, using one type of MRP, the Beam + telepresence robot. Future work should be done using a variety of tasks and other brands of MRPs to determine the potential effect of the task type and MRP features on participants' comfortability with initiating social interactions. In addition, future work is needed to see how well the MRP functions in a real-world educational setting.

\section{DISCUSSION}

In this exploratory study, we interviewed ten undergraduate students to better understand their views on MRP systems for personal and interpersonal use.

Regarding personal aspects of MRP use, we found that students saw benefits to the MRP, including mobility, physical embodiment, and independence of movement. All of them drove the robot easily and most wished that it moved faster so they could get tasks done more efficiently. However, even though driving was reported as easy, some participants mentioned that it was not easy for them to multitask (e.g., talking, reading task instructions) and navigate the MRP at the same time. Autonomous MRPs could be a solution to this problem (e.g., [3]) however it should be taken into account that fully autonomous systems can result in higher workload compared to manual MRPs due to users' lack of trust in automated system especially when the underlying mechanism of automation is not clear to users [12]. Therefore, semi-autonomous MRPs that allow some level of control by the user or assisted driving features (e.g., [17]) might be better options.

Regarding interpersonal aspects of MRP use, we found that the majority of our participants were hesitant to approach people and engage in social interactions. Among the three reasons we found on this issue only one of them, the novelty of robot might become less of an issue by repeated exposure and practice using the MRP. Alleviating the other two points, self-presentational concerns and difficulty interpreting nonverbal signals, will require further research and design solutions.

One consideration to take into account in designing MRPs for use by college students is that when students use MRPs they might find themselves in an environment where they do not know people who are collocated with the MRP. Initiating conversations with strangers could be harder than talking to friends or people they know. Therefore, it is important to consider conversation starters and ice-breakers in the design of MRPs that are supposed to be used in settings where people do not know each other. As mentioned earlier in this paper, MRPs could provide an opportunity to students who attend classes remotely a chance to meet classmates outside class and it is important to consider ways to facilitate initiation of conversation for these students especially in the initial steps of the MRP use when the novelty of the robot mediated communication could act as a barrier to social interactions as well. Related to this, researchers has suggested an MRP system that propose salutations and greetings [5] and we suggest conversation starters that appear on screen (e.g., "I'm looking for room 14") that could facilitate initiation of interaction by locals. For those with high self-presentational concerns, feedback on loudness of voice (e.g.,[11]) and an option to use a static selfie image might be appealing.

\section{ACKNOWLEDGMENTS}

We thank Jeff Rzeszotarski and Wen Duan for their helpful comments on previous drafts of this paper. This work was supported in part by National Science Foundation award IIS-1563705. 


\section{REFERENCES}

[1] Jacob T. Biehl, Daniel Avrahami, and Anthony Dunnigan. 2015. Not Really There: Understanding Embodied Communication Affordances in Team Perception and Participation. In Proceedings of the 18th ACM Conference on Computer Supported Cooperative Work \& Social Computing (CSCW '15). ACM, New York, NY, USA, 1567-1575. https://doi.org/10.1145/2675133. 2675220

[2] Elizabeth Cha, Qandeel Sajid, and Maja J. Matarić. 2016. Enabling Access to K-12 Education with Mobile Remote Presence. In AAAI Spring Symposium Series.

[3] Akansel Cosgun, Dinei A. Florencio, and Henrik I. Christensen. 2013. Autonomous person following for telepresence robots. In 2013 IEEE International Conference on Robotics and Automation. 4335-4342. https://doi.org/10.1109/ICRA.2013.6631191

[4] Naomi T. Fitter, Yasmin Chowdhury, Elizabeth Cha, Leila Takayama, and Maja J. Matarić. 2018. Evaluating the Effects of Personalized Appearance on Telepresence Robots for Education. In Companion of the 2018 ACM/IEEE International Conference on Human-Robot Interaction (HRI '18). ACM, New York, NY, USA, 109-110. https://doi.org/10.1145/3173386. 3177030

[5] Hideaki Kuzuoka, Yuki Kodama, Jianfeng Xu, Emi Myodo, Etsuko Harada, and Hirotaka Osawa. 2018. Telepresence Robot's Salutations to Trigger Informal Conversation with Teleworkers. In Companion of the 2018 ACM Conference on Computer Supported Cooperative Work and Social Computing (CSCW '18). ACM, New York, NY, USA, 233-236. https: //doi.org/10.1145/3272973.3274063

[6] Min Kyung Lee and Leila Takayama. 2011. "Now, I Have a Body": Uses and Social Norms for Mobile Remote Presence in the Workplace. In Proceedings of the SIGCHI Conference on Human Factors in Computing Systems (CHI '11). ACM, New York, NY, USA, 33-42. https://doi.org/10.1145/1978942.1978950

[7] John Lofland and Lyn H. Lofland. 1995. Analyzing Social Settings: a Guide to Qualitative Observation and Analysis (3rd. ed.) Wadsworth Publishing Company, Belmont, CA, USA

[8] Carman Neustaedter, Gina Venolia, Jason Procyk, and Daniel Hawkins. 2016. To Beam or Not to Beam: A Study of Remote Telepresence Attendance at an Academic Conference. In Proceedings of the 19th ACM Conference on Computer-Supported Cooperative Work \& Social Computing (CSCW '16). ACM, New York, NY, USA, 418-431. https://doi.org/10.1145/2818048. 2819922

[9] Veronica Ahumada Newhart and Judith S. Olson. 2017. My Student is a Robot: How Schools Manage Telepresence Experiences for Students. In Proceedings of the 2017 CHI Conference on Human Factors in Computing Systems (CHI '17). ACM, New York, NY, USA, 342-347. https://doi.org/10.1145/3025453.3025809

[10] Veronica Ahumada Newhart, Mark Warschauer, and Leonard S. Sender. 2016. Virtual inclusion via telepresence robots in the classroom: An exploratory case study. The International Journal of Technologies in Learning 23, 4 (2016).

[11] Andreas Paepcke, Bianca Soto, Leila Takayama, Frank Koenig, and Blaise Gassend. 2011. Yelling in the Hall: Using Sidetone to Address a Problem with Mobile Remote Presence Systems. In Proceedings of the 24th Annual ACM Symposium on Use Interface Software and Technology (UIST '11). ACM, New York, NY, USA, 107-116. https://doi.org/10.1145/2047196.2047209

[12] Raja Parasuraman, Thomas B. Sheridan, and Christopher D. Wickens. 2008. Situation Awareness, Mental Workload, and Trust in Automation: Viable, Empirically Supported Cognitive Engineering Constructs. Journal of Cognitive Engineering and Decision Making 2, 2 (2008), 140-160. https://doi.org/10.1518/155534308X284417 arXiv:https://doi.org/10.1518/155534308X284417

[13] Irene Rae and Carman Neustaedter. 2017. Robotic Telepresence at Scale. In Proceedings of the 2017 CHI Conference on Human Factors in Computing Systems (CHI '17). ACM, New York, NY, USA, 313-324. https://doi.org/10.1145/3025453.3025855

[14] Brett Stoll, Samantha Reig, Lucy He, Ian Kaplan, Malte F. Jung, and Susan R. Fussell. 2018. Wait, Can You Move the Robot?: Examining Telepresence Robot Use in Collaborative Teams. In Proceedings of the 2018 ACM/IEEE International Conference 
on Human-Robot Interaction (HRI '18). ACM, New York, NY, USA, 14-22. https://doi.org/10.1145/3171221.3171243

15] Suitable Technologies. 2019. Beam software. Retrieved Aug 17, 2019 from "https://suitabletech.com"

[16] Leila Takayama and Helen Harris. 2013. Presentation of (Telepresent) Self: On the Double-edged Effects of Mirrors. In Proceedings of the 8th ACM/IEEE International Conference on Human-robot Interaction (HRI '13). IEEE Press, Piscataway, NJ, USA, 381-388. http://dl.acm.org/citation.cfm?id=2447556.2447684

[17] Leila Takayama, Eitan Marder-Eppstein, Helen Harris, and Jenay M. Beer. 2011. Assisted driving of a mobile remote presence system: System design and controlled user evaluation. In 2011 IEEE International Conference on Robotics and Automation. 1883-1889. https://doi.org/10.1109/ICRA.2011.5979637

[18] Fumihide Tanaka, Toshimitsu Takahashi, Shizuko Matsuzoe, Nao Tazawa, and Masahiko Morita. 2014. Telepresence Robot Helps Children in Communicating with Teachers Who Speak a Different Language. In Proceedings of the 2014 ACM/IEEE International Conference on Human-robot Interaction (HRI '14). ACM, New York, NY, USA, 399-406. https: //doi.org/10.1145/2559636.2559654

[19] Katherine M. Tsui, Munjal Desai, Holly A. Yanco, and Chris Uhlik. 2011. Exploring Use Cases for Telepresence Robots. In Proceedings of the 6th International Conference on Human-robot Interaction (HRI '11). ACM, New York, NY, USA, 11-18 https://doi.org/10.1145/1957656.1957664 